\title{
Electron paramagnetic resonance and mass spectrometry: Useful tools to detect ultraviolet light induced skin lesions on a molecular basis - A short review
}

\author{
Ulrike Hochkirch $^{\mathrm{a}}$, Werner Herrmann ${ }^{\mathrm{b}}$, Reinhard Stößer ${ }^{\mathrm{a}}$, Hans-Hubert Borchert ${ }^{\mathrm{b}}$ and \\ Michael W. Linscheid ${ }^{\mathrm{a}, *}$ \\ ${ }^{a}$ Department of Chemistry, Humboldt-Universität zu Berlin, Brook-Taylor-Str. 2, 12489 Berlin, \\ Germany \\ ${ }^{\mathrm{b}}$ Department of Pharmacy, Freie Universität Berlin, Kelchstr. 31, 12169 Berlin, Germany
}

\begin{abstract}
Ultraviolet radiation is considered responsible for sunburning, premature skin aging, and cancerogenesis through the production of free radical species. Therefore, the favoured possibility for direct detection of unpaired electrons - electron paramagnetic resonance spectroscopy - is predestinated for detection and structural and dynamic analysis of this kind of molecules. However, many of UV induced radicals in skin have a short lifetime at ambient conditions and possibilities for stabilisation or transformation into definite para- or diamagnetic products have to be found. On the other hand, diamagnetic products, potentially also originated by reporter molecules, which are not detectable by EPR, are target molecules for mass spectrometric analysis. In this review, potentials and limitations of both spectroscopic methods are reviewed, and the effect of ultraviolet radiation on human skin is discussed in particular. Suitable combinations of both techniques result in detailed information about photoproducts and processes taking place within skin during and after irradiation. The literature is viewed from a recent perspective; historical aspects were not in the scope of this paper.
\end{abstract}

Keywords: EPR/ESR, MS, UV light, skin, free radicals, imaging, quantification
Abbreviations
AP atmospheric pressure,
APCI atmospheric pressure chemical ionisation,
CAT-1 4-trimethylamino-2,2,6,6-tetramethylpiperidine-1-oxyl,
COLIPA The European Cosmetic Toiletry and Perfumery Association,
cw continuous wave,
DESI desorption electrospray ionisation,
DMPO 5,5-dimethyl-1-pyrroline-N-oxide,
DNA deoxyribonucleic acid,
DPPH 1,1-diphenyl-2-picrylhydrazyl,
EPR electron paramagnetic resonance,

\footnotetext{
${ }^{*}$ Corresponding author: Michael W. Linscheid, Department of Chemistry, Humboldt-Universität zu Berlin, Brook-TaylorStr. 2, 12489 Berlin, Germany, Tel.: +49 302093 7575; Fax: +49 302093 6985; E-mail: m.linscheid@ chemie.hu-berlin.de.
} 


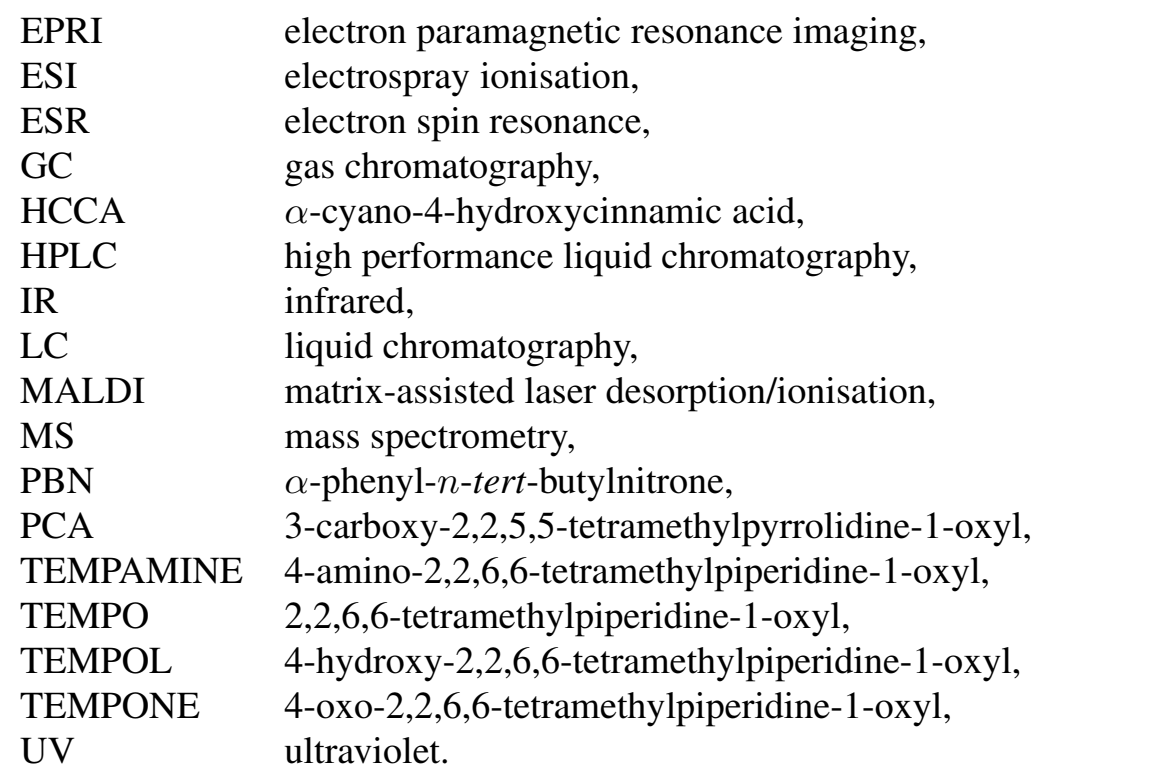

\section{Introduction}

Electron paramagnetic resonance and mass spectrometry are two different spectroscopic methods, which can complement one another: With the very specific EPR it is possible to detect radical species in a direct way. The unpaired electron gives response to the employed microwave energy depending on its chemical and physical vicinity. MS is more general and allows to measure a wide range of molecules, provided they are ionisable. This includes radicals too, even though these species develop a chemistry of their own within the mass spectrometer depending mainly on the kind of molecule and ionisation technique [1]. For both spectroscopic methods imaging techniques have been developed requiring different, more or less complex sample preparation. Of course, for detecting UV light induced molecular changes within skin, sample preparation should be as simple as possible considering the fact that some UV initiated chemical reactions are very fast and primary photoproducts might not be stable.

\section{Interaction between skin and UV radiation}

\subsection{Human skin}

The skin forms the outer barrier of the human body to the environment and protects the organism against chemical, physical, and microbiological stress. Its composition is described in many textbooks and is not within the scope of this article. Obviously, the stratum corneum with a thickness of 15 to 20 cell layers is that part of the skin, which is mostly affected by UV light. Its structure and function is reviewed in detail by Marks [2].

When experiments on human skin biopsies are carried out one has to consider the biological conditions of the object of investigation. It depends on various factors like origin (sex and age of donor, body area), pre-treatment with medicals and cosmetics and viability after surgery. Wester at al. found that viability, determined by anaerobic metabolism (conversion of glucose to lactate), decreases in the first eighteen 
hours by $70 \%$ but then remains constant until day 8 [3]. Moll verifies these results and uses skin biopsies from day 2 until day 5 after surgery only [4]. Thus, experiments should be carried out within this time for the sake of reproducibility and comparability.

\subsection{Sun light and its $U V$ part}

The types of optical radiations reaching the skin are UV light, visible light, and IR radiation. Some of them have beneficial properties such as the anti-rickets action of UV, which transforms 3dehydrocholesterol (provitamin $\mathrm{D}_{3}$ ) into vitamin $\mathrm{D}_{3}$, the anti-depressive effect of visible light, the caloric effect of IR, and the global anti-inflammatory and anti-allergic effects. Further the human's well-being is significantly dependent on sunshine, regardless the problems suntanning is a symbol of health in our society.

UV light is an important part of sun light with wavelengths between 100 and $400 \mathrm{~nm}$ and is divided into three regions: UVC (100-280 nm), UVB (280-320 nm) and UVA (320-400 nm) [5]. These definitions date back to the early part of the last century based on a combination of physical properties and biological effects of the spectral parts [6]. The corresponding energies of ultraviolet light reach from $3.1 \mathrm{eV}(400 \mathrm{~nm})$ to $12.4 \mathrm{eV}(100 \mathrm{~nm})$, and the ionisation energy of water $(12.6 \mathrm{eV})$ marks the border to ionising radiation. Most important in this context, the excitation of water molecules at $7 \mathrm{eV}$ followed by direct decomposition into ${ }^{\bullet} \mathrm{OH}$ radical and $\mathrm{H}^{\bullet}$ radical has been described [7], which react as aggressive agents in biological tissues. For comparison the energy of hydrogen bond $(0.1-0.3 \mathrm{eV})$, carbon-carbon bond with approximately $3.6 \mathrm{eV}$ (in ethane) and ionisation energy of vitamin $\mathrm{C}$ with $11.8 \mathrm{eV}$ are mentioned in Fig. 1.

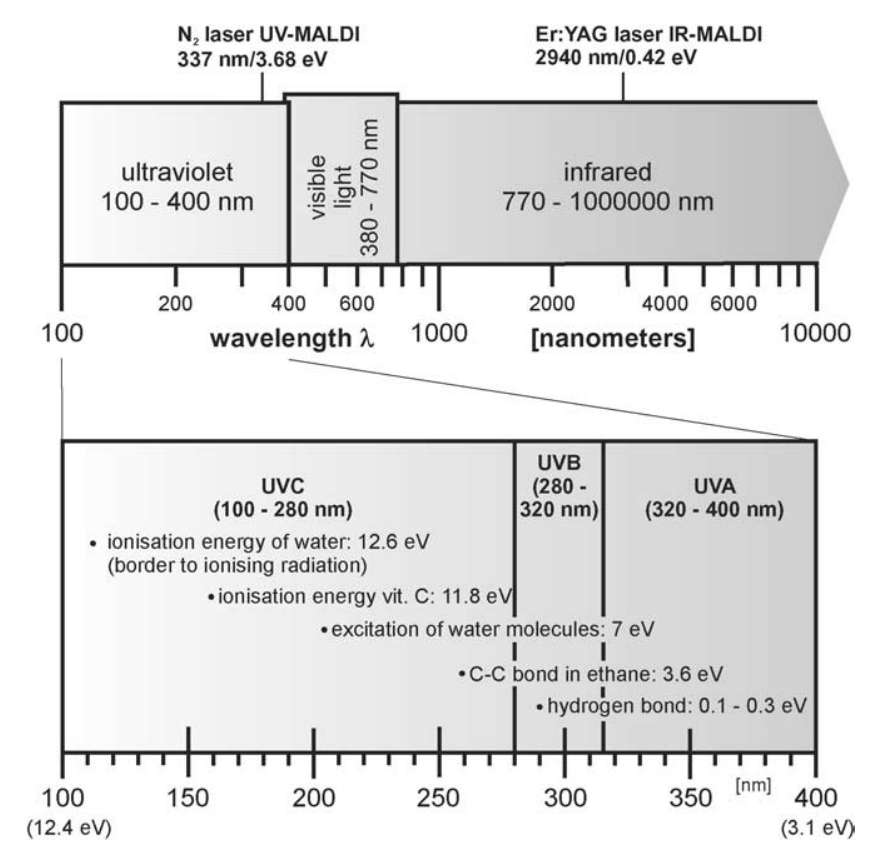

Fig. 1. UV light in the electromagnetic spectrum. For comparison the binding energies of some compounds and selected laser energies for MALDI excitation are given. 

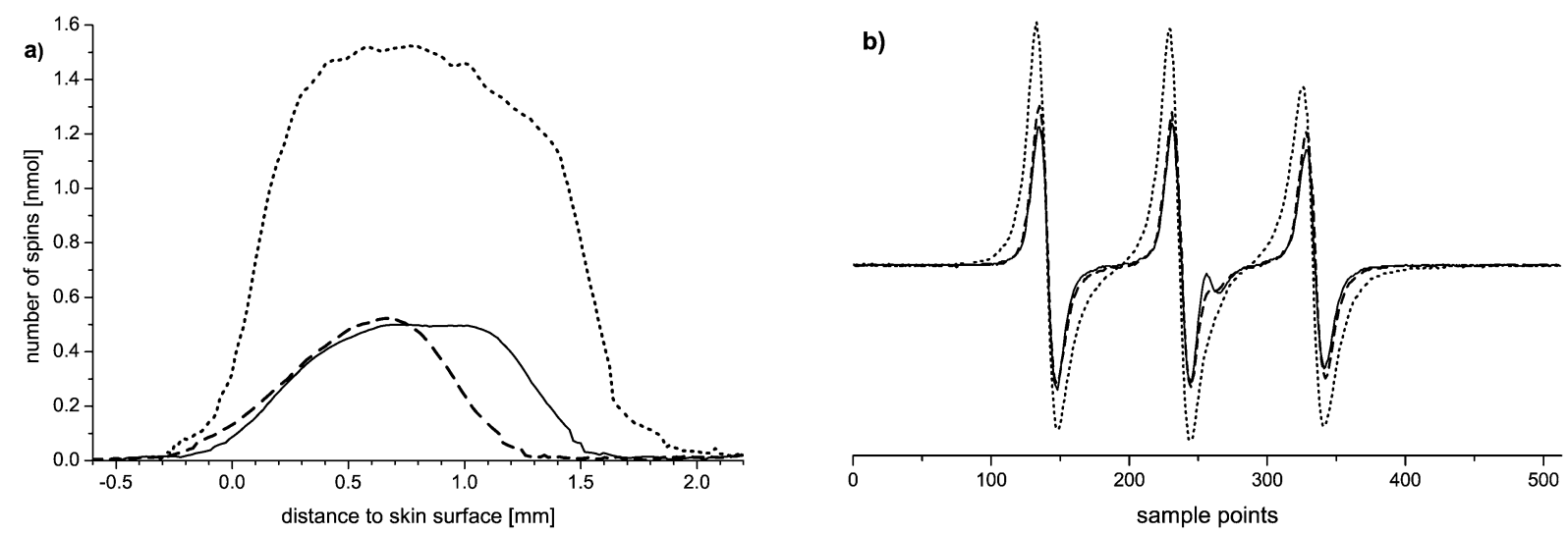

Fig. 2. Radical concentration in human skin. Spin probe CAT-1 (see Scheme 1) acts as a reporter molecule. Skin biopsy was soaked in $25 \mathrm{mM}$ CAT-1 solution for $30 \mathrm{~min}$. Straight line: no irradiation, dashed line: UVC irradiation after incubation with spin probe, dotted line: UVC irradiation before incubation with spin probe. (a) The spin concentration over the skin layers resulted from an imaging experiment and is calculated from the second integral of the EPR signal and normalised versus an internal standard. (b) Overall EPR signal from the cw experiment. The comparison between integrals and amplitudes shows clearly that the exclusive analysis of amplitudes leads to an underestimation of the radical concentration.

\subsubsection{Negative effects of UV light on human skin}

It is clearly established that the exposure of human skin to ultraviolet light can cause sunburning (UVB), skin photoaging (UVA), immunosuppression, and skin cancer. UVC is the most energy-rich and hazardous kind of UV light, which is almost completely absorbed by the ozonosphere. However, the loss of ozone in the stratosphere increases the potential risk of UVC light exposure. The shortest wavelengths of the ultraviolet spectrum are reflected and scattered by the outermost layers of skin. But due to a content of $15 \%$ water in the stratum corneum [2] and a total iron content of $62.5 \mathrm{ppm}$ in the epidermis [8] UVC may lead to the formation of ${ }^{\bullet} \mathrm{OH}$ and $\mathrm{H}^{\bullet}$ radicals on the skin surface. Although the light should not enter the skin we found the stratum corneum affected and changes in the reducing potential of skin (e.g. enzymatic systems) are evident (Fig. 2). UVB is considered responsible for a set of damages like sunburn, which is the inflammatory response of the outer layers of the epidermis generating so-called 'sunburn-cells'. These are keratinocytes, that undergo apoptosis (programmed cell death) caused by irreparable UV induced DNA damages. The maximum absorption of DNA ranges from 245 to $290 \mathrm{~nm}$ [9] resulting in mutagenic photoproducts predominantly in the form of cyclobutane pyrimidine dimers and pyrimidine (6-4) pyrimidone photoproducts [10-12]. UVA is the UV radiation of the lowest energy, but it reaches deeper, living layers of the skin. It is suspected to play an important role in photoaging and the appearance of wrinkles. UVA is believed to induce oxidative lesions in membrane lipids and amino acids via an indirect route, the photosensitisation of endogenous chromophores [11,13]. Whereas in type I photosensitisation excited sensitisers transfer charges directly to DNA, in type II oxygen is activated first. However, both routes mainly lead to an oxidation of the guanine base in the DNA resulting in 8oxo-7,8-dihydroguanine as the main oxidative DNA damage [14]. An extensive survey of the short- and long-term effects of UV light on human skin is given by Matsumura and Ananthaswamy [11].

\subsection{Sun protection}

The importance of sunscreens to avoid sunburn and other expected damages by UV light is under increasing discussion. Whereas the declaration of the sun protection factor of UVB determined by the 
COLIPA method [15] is an internationally accepted standard, the classification of UVA protection is still nonuniform. Most manufacturers use the Australian Standard [16], which regulates that a sunscreen has to reduce the transmission between 320 and $360 \mathrm{~nm}$ by at least $90 \%$. Further differentiation is not required with the consequence that at very high sun protection factors an adequate UVA protection is not necessarily given. A very new but national standard (Germany) is based on an in vitro method, which considers the results of the in vivo sun protection factor for UVB [17].

Another approach to a standardised determination of UV originated free radicals in skin is the 'Integrated Sun Protection Factor' using EPR imaging measurements of trapped radicals, which is still on an early experimental stage [18].

The up to now inconsistent and insufficient methods to estimate the UV induced skin damages and the fact that the determination of the sun protection factor is still an in vivo method on human beings demands the development of new and complementary techniques.

\subsection{The use of spin probes observing UV damaging effects}

To overcome the problem that UV induced radicals in biological tissue reach a low steady state concentration, the application of spin traps and spin probes is common. A spin trap is a diamagnetic compound containing a nitroso or nitrone function, which reacts with primary radicals readily to form more stable radical adducts (see Section 3). A spin probe, which is a stable free radical, has the advantage that its chemical identity during the experiment is known. TEMPOL, as one example, is used extensively as a scavenger in polymerisation reactions.

\subsubsection{Behaviour of spin probes in human skin}

Mostly, the used spin probes are nitroxide radicals with five- ore six-membered alicyclic rings attached, which in solution usually give a three line spectrum in the EPR (Scheme 1). Under laboratory conditions they are very stable and their metabolic chemistry within living tissue is essentially limited to the reduction to the corresponding hydroxylamine [19]. Six membered rings are less stable and only they are able to undergo a further reduction to the secondary amine, however this occurs to a very low

a)<smiles>CCC1CC(C)(C)N(O)C(C)(C)CC1[N+](C)(C)C</smiles>

c)<smiles>CC1(C)CCCC(C)(C)N1O</smiles>

b)<smiles>[R]C1CC(C)(C)N(O)C(C)(C)C1</smiles>

d)<smiles>CC1(C)CC(C(=O)O)C(C)(C)N1[O]</smiles>

Scheme 1. Selection of spin probes used for EPR experiments. (a) CAT-1; (b) TEMPONE and TEMPAMINE; (c) TEMPO; (d) PCA. 
rate [20,21]. Small molecules such as the vitamins A,C,E and glutathione or enzymatic systems, whose availability and activity depend on the location, are the main antioxidants or radical scavengers of the skin $[22,23]$. When selecting a nitroxide spin probe it is not only important, whether it enters the cell or not (usually uncharged lipophilic molecules enter cells more easily than charged), also toxicity plays an important role. Some nitroxide radicals are reported to be not or slightly irritant [24], and are already investigated on human skin in vivo [25,26].

In our experiments, human skin biopsies of healthy volunteers are incubated with an aqueous solution of spin probe CAT-1 and its concentration over skin thickness is visualised using an EPR imaging technique [27]. According to its very small n-octanol/water partition coefficient (i.e., it is readily soluble in water) [21] it is dominantly found in water containing domains, i.e. the deeper skin layers above subcutis. After a certain incubation period, the radical concentration reaches a steady-state level. Figures 3 and 4 make clear the enrichment of some spin probes in the hydrophilic or lipophilic vicinity in model experiments and in skin biopsies, which corresponds to the Nernst distribution law [4,28-30].

The impossibility of the direct detection of diamagnetic compounds by means of EPR is disadvantageous, but can be compensated by the use of mass spectrometry, which gives complementary information about the amount of the stable free radical, its corresponding hydroxylamine and eventually further biodegraded species. A charge is the only requirement of mass spectrometry on the molecule that is fulfilled by the used spin probe radical as well as by its corresponding hydroxylamine. Neutral spin probes can be charged within the ion source, however, the precise mechanisms are dependent on the ionisation technique, the nature of the molecule, and the analysing conditions and are still under investigation [1]. A large amount of molecules is accessible by MS, but there is one disadvantage, which can be compensated by non-destructive EPR imaging: the biopsies have to be cut in small slices if a tomographic picture in the original sense is required (gr. tomos - slice). The problem of sample preparation for MS experiments like the extraction of the spin probe is minimised by the use of MALDI technique directly from thin human skin biopsies in special MALDI targets (Fig. 5). Figure 6 shows the results of an EPR tomography (a) and a MALDI-MS examination (b) of a skin biopsy incubated in spin probe solution for 30 min. Whereas Fig. 6a illustrates the concentration profile of the spin probe over the skin layers non-

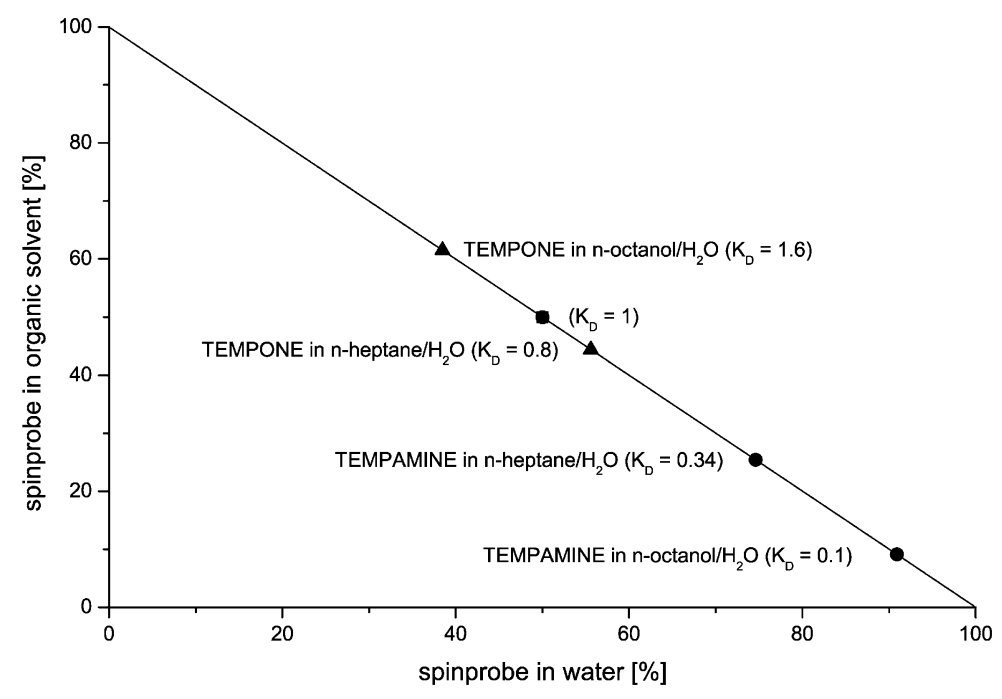

Fig. 3. Solubility of two selected spin probes in water and in organic solvents. 


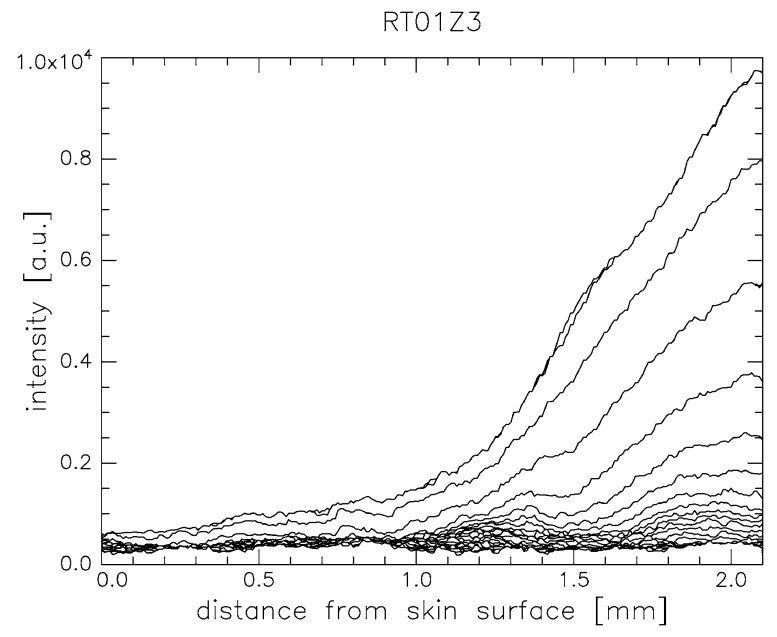

(a)

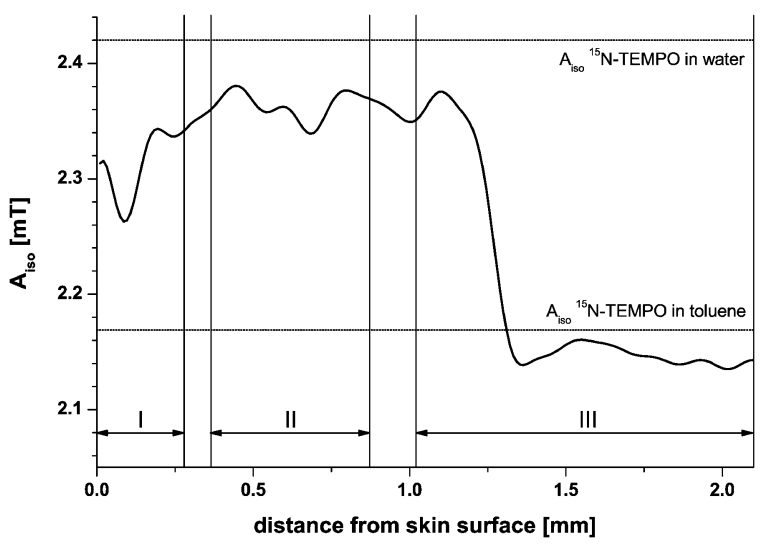

(b)

Fig. 4. Penetration of the spin probe ${ }^{15} \mathrm{~N}-\mathrm{TEMPO}$ in excised human abdominal skin. (a) Distribution profile, after 30 min of incubation with $30 \mathrm{mM}{ }^{15} \mathrm{~N}$-TEMPO in a microemulsion; (b) the assignment of hyperfine splitting constants $A_{\text {iso }}$ to the skin layers (I: epidermis, II: upper dermis, III: lower dermis; for comparison the values of $A_{\text {iso }}$ of ${ }^{15} \mathrm{~N}$-TEMPO in the pure solvents water and toluene are marked representing the extreme cases).

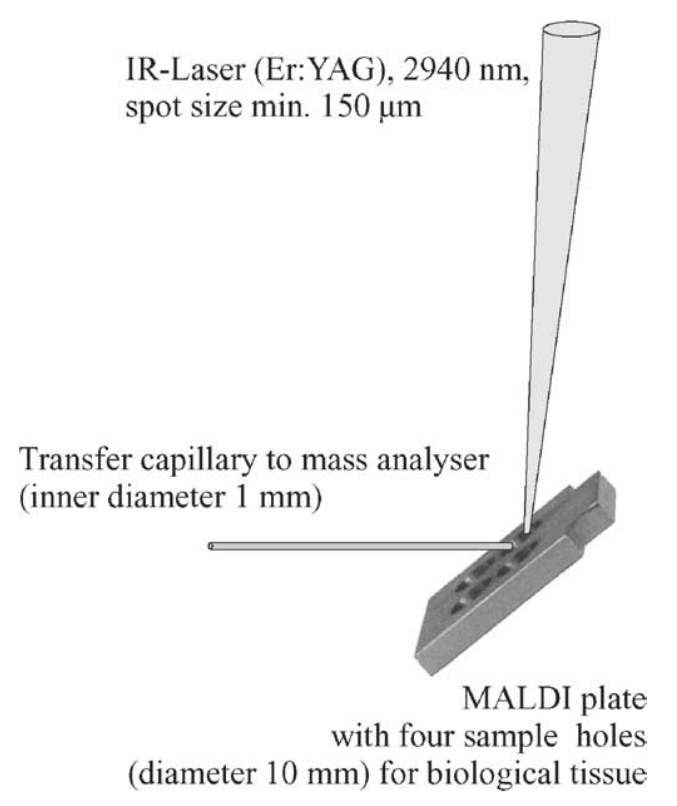

Fig. 5. MALDI target for analysis of small skin biopsies.

invasive Fig. $6 \mathrm{~b}$ stands for the proportion between nitroxide radical and its corresponding hydroxylamine on the skin surface.

Somewhat comparable spatial information by means of MALDI-MS might be obtained by examining microtome slices, but requires the application of an invasive technique. 


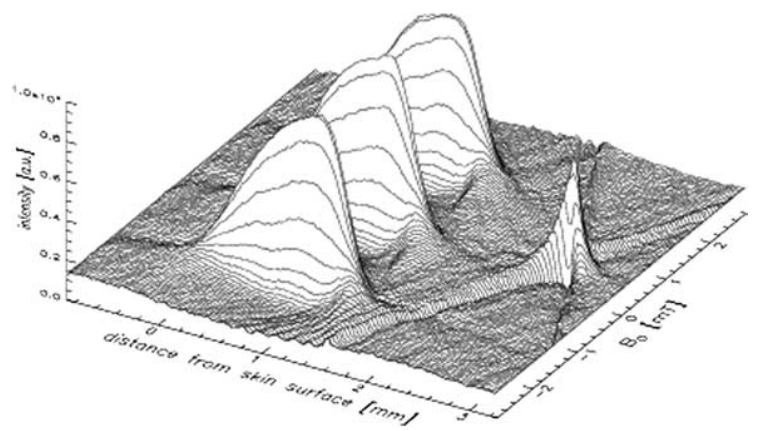

(a)

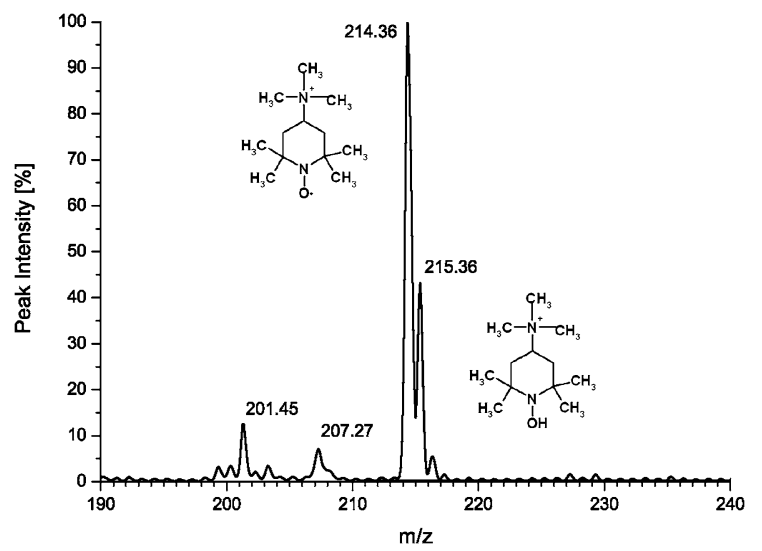

(b)

Fig. 6. Distribution of the spin probe CAT-1 in a human skin biopsy. Without irradiation, the tissue was incubated for 30 min with a $25 \mathrm{mM}$ aqueous solution of CAT- 1 at $32^{\circ} \mathrm{C}$. Results of the (a) EPR imaging experiment (the single peak at the right is due to the internal standard DPPH; its spin concentration was determined versus a second DPPH crystal, whose quantification was done by UV-VIS spectroscopy [82]) and (b) MALDI-MS experiment (glycerol was used as matrix on the skin surface; nearly half of the free radical is reduced to the corresponding hydroxylamine; other secondary products are not detectable).

\subsubsection{Influence of UV light}

Some research groups already found a nitroxide (TEMPOL) to be a good protector against ultraviolet irradiation [31-33]. Herrling et al. used nitroxides and found them suitable to detect UV generated radicals in human skin biopsies [34]. Our work uses the nitroxides' relatively simple chemistry and almost non-toxic nature to observe quantitatively UV light induced changes in human skin. Thereby CAT-1 acts as a molecular marker, which gives information about processes taking place inside the sample after UV irradiation. After a steady-state level of spin probe concentration within the biopsy is reached, it is irradiated with different UV wavelengths. UVC ${ }^{1}$ causes no or a tiny decrease of radical concentration assuming that UVC does not penetrate the skin. From model experiments in water, it is known that CAT-1 changes very fast to an EPR silent species at this wavelength. Surprisingly, almost all reducing properties of the tissue disappear, when the skin biopsy is irradiated before preconditioning with spin probe. Obviously, the UVC degrades a large amount of reducing agents of the skin, but the mechanism is unknown up to now (Fig. 2).

However, first experimental results show that light with lower energy, not affecting CAT-1 in aqueous solution, has a remarkable effect on the spin probe inside the skin biopsy, too.

If the influence of UV radiation on this system is monitored by MALDI mass spectrometry, the use of the most common excitation source in this technique - nitrogen laser with a wavelength of $337 \mathrm{~nm}$ in the UVA range - is not reasonable. Excitation with an UV laser in vacuum MALDI systems usually requires a matrix, forming analyte-matrix co-crystals. This takes time and allows the free radical/hydroxylamine system to undergo further conversions obscuring its original nature and concentration inside the skin. To avoid competing effects between mass spectrometric excitation and previous ultraviolet irradiation of sample we use an infrared Er:YAG laser $(\lambda=2940 \mathrm{~nm})$. Model experiments show a clear increase of hydroxylamine versus nitroxide radical on using the nitrogen laser, which is due to the higher energy of light or the use of HCCA as a matrix (Fig. 7). Another advantage of IR-MALDI is the option of the direct analysis of original wet tissue when glycerol is applied as a matrix.

\footnotetext{
${ }^{1}$ KrF-Laser Compex100, wavelength: $248 \mathrm{~nm}$, energy: $150 \mathrm{~mJ}$, spot diameter: $8 \mathrm{~mm}$.
} 

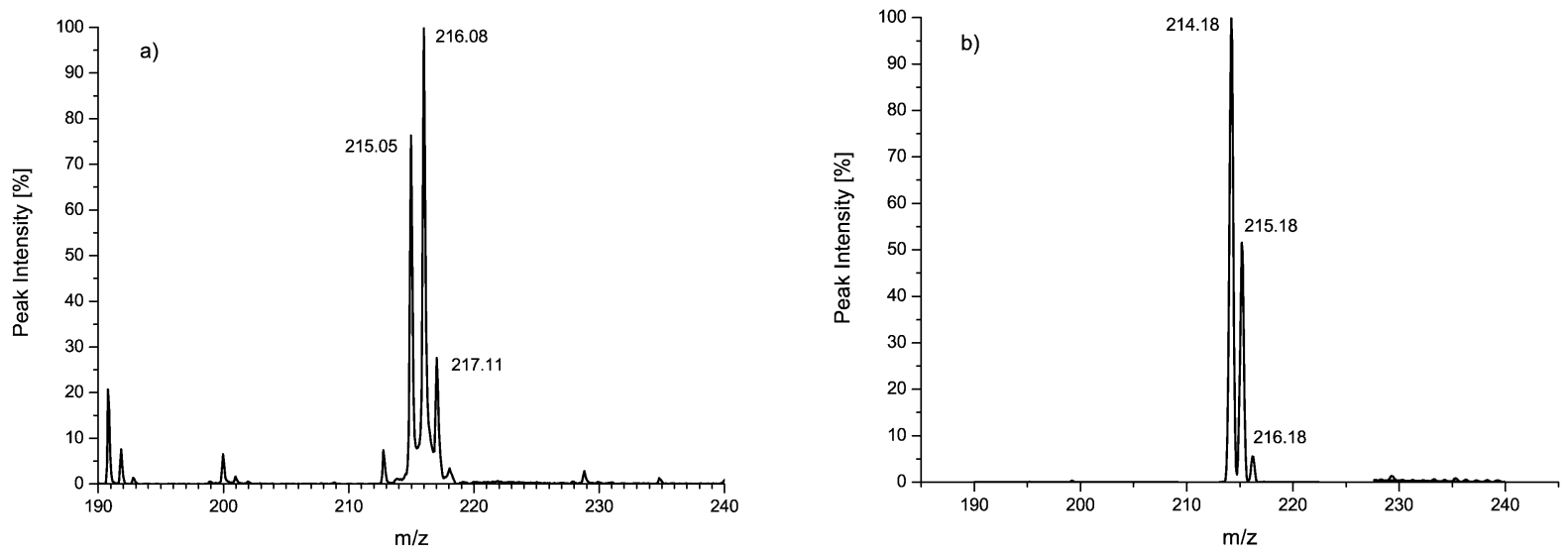

Fig. 7. MALDI-MS of CAT-1 in water. (a) Excitation with a nitrogen laser at $337 \mathrm{~nm}$ in a MALDI Time of Flight MS (matrix: HCCA); (b) excitation with an Er:YAG laser at $2940 \mathrm{~nm}$ in a AP-MALDI Ion Trap MS (matrix: glycerol); excitation with UV laser in a vacuum system gives much less free radical but more hydroxylamine than via excitation with IR laser.

\section{Electron paramagnetic resonance spectroscopy}

EPR with its unique feature for the direct detection of paramagnetic species as produced by UV light in human skin should be very helpful to answer some of the questions concerning UV induced skin lesions. As mentioned already, the largest problem to detect these species is their mostly very short lifetime at physiological conditions, which makes a measurement very difficult. Different approaches to overcome this problem are described in the following sections.

\subsection{Direct detection of $U V$ generated radicals in skin}

Jurkiewicz and Buettner used the ascorbate free radical present at a very low steady-state level in unexposed skin to detect UV induced radical formation. Increase of signal intensity, measured as height of signal amplitude of $\mathrm{cw}$ EPR measurements, indicates that during irradiation free radical oxidative stress occurs [35,36]. However, Haywood et al. showed that a significant increase of ascorbate radical concentration is detectable during irradiation only. After switching off the light source, the radical concentration almost declines to the original amount before treatment [37]. Such an experiment will be difficult to be carried out in an arrangement for 1D EPR imaging employing a z-gradient, because the skin surface has to face the gradient coils for measurement (see Fig. 8).

\subsection{Stabilising short-lived radicals}

Human skin is a living system with only few possibilities for stabilising radicals, if physiological conditions should be preserved. The use of spin traps is the most frequently applied method for this purpose $[18,36,38]$. It involves the reaction of a free radical with a diamagnetic trapping compound resulting in a longer living, EPR detectable species. Hereby, the lifetime of the product depends on the kind of the original radical, the spin trap, the solvent, and other parameters. For example, Zastrow et al. found PBN-adducts to provide a stable EPR signal during a time of about 60 min after UV irradiation of skin biopsies [39]. The time development of spin-trapped ${ }^{\bullet} \mathrm{OH}$ radicals with DMPO as trap was studied by Yanagida et al. [40]. The half-life of ${ }^{\bullet} \mathrm{DMPO}-\mathrm{OH}$ was determined up to a maximum of 10 min depending on the starting conditions. However, the quantification of UV generated radicals should be possible if 


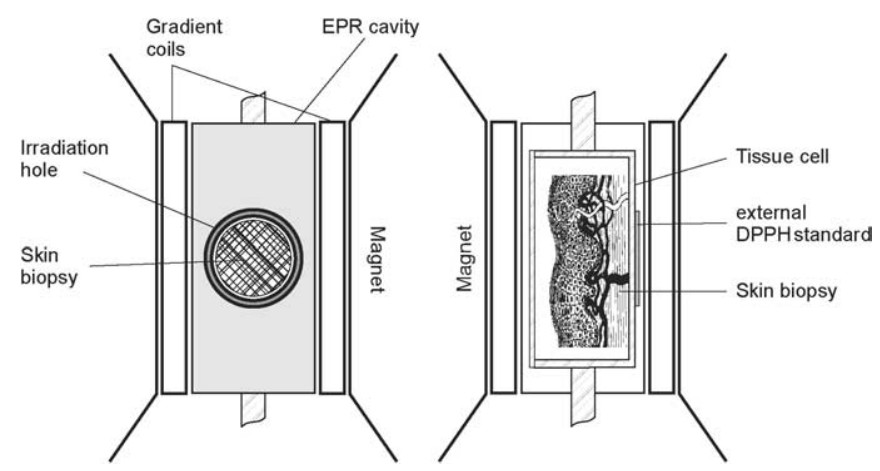

Fig. 8. Arrangement of the skin biopsy in the EPR cavity. Left: The stratum corneum of the skin is seen through the irradiation hole of the $\mathrm{TE}_{102}$ resonator. For the tomography measurement, the sample has to be turned by 90 degrees. Right: section through the resonator for the visualisation of the arrangement during the EPR measurement; the stratum corneum is now facing the gradient coils. (Sketch is not scaled.)

the time dependence of the stability of the trap-adducts is considered. Yanagida et al. mentioned the fact, that the half-life of ${ }^{\bullet} \mathrm{DMPO}-\mathrm{OH}$ gets shorter, when the reaction proceeds indicating a complex chemistry of the spin trapping mechanism [40]. The application of spin probes and hydroxylamines is another possibility for stabilising radicals in human tissue as already mentioned in Section 2.4. The benefit of both kinds of molecules is their simple chemistry. Thereby six-membered ring radicals react faster than five-membered rings, whereas most of the bioreduction occurs within the cells. Furthermore, the structure and properties of the nitroxide radical, the influence of oxygen, temperature, $\mathrm{pH}$ value and whether the reduction is enzymatic or not have to be considered, when nitroxide radicals should be observed in skin [22]. This should be valid for the hydroxylamines, too, but up to now not much is known about them within living tissues and further investigations are required.

Dikalov et al. made one of the first attempts concerning the comparison of spin trapping and the use of hydroxylamines for detecting superoxide quantitatively in biological tissues [41]. They found hydroxylamines with various lipophilicity and cell permeability to be more sensitive than nitrone spin traps.

\subsection{Quantification}

EPR spectroscopy is not a routine method for the quantification of radicals. However, a quantification is possible and Yordanov et al. did pioneering work in this field [42-45]. In addition to an appropriate internal standard like $\mathrm{Mn}^{2+}$ or DPPH a proper sample positioning within the resonator is one of the most important requirements for quantification.

A cw measurement supplies a spectrum, which reflects all contributions of the spectral response (radicals, paramagnetic ions, etc.). The analysis of the spectrum reveals the different contributors and the double integration provides information about the respective quantities. Moreover, the usage of special equipment (double resonator) allows the determination of the spin concentration compared to a reference sample.

In the case of EPR imaging the quantification will be even somewhat easier, because sample and reference will be measured simultaneously. Due to the spatial separation of sample and reference, it will not be necessary to analyse the spectra concerning the individual contributions. However, if statements with respect to spatial distribution phenomena or alike are needed only EPR imaging can provide such kind of quantitative information $[18,28,46,47]$. 


\section{Mass spectrometry}

MS is a widely used analytical method and is applied to a large array of biological questions. Among others, the method is characterised by different ionisation techniques such as electron impact, electrospray ionisation, or matrix-assisted laser desorption/ionisation resulting in more or less fragmented molecular ions. Even though, MS is one of the most important techniques, there are only few attempts for a direct analysis of living material.

As discussed before, MALDI-MS not always needs a major sample preparation (e.g., extraction techniques) to detect compounds like biomolecules in a mixture, but the co-crystallisation with the matrix is essential. Thus, it should be possible to detect UV generated molecular changes in skin with MALDIMS, too. Moreover, for that purpose one can use tissue sections or even complete tissues.

\subsection{Analysis of model compounds and extracted material}

There is a large spectrum of molecules on and in skin which are affected by UV light and only some examples are presented here. Mudiyanselage et al. investigated squalene, a skin surface lipid, and found it to be very sensitive against UV irradiation. Squalene monohydroperoxide photoproducts were identified via APCI-MS [48]. Fatty acids, cholesterol, and phospholipids are other components in stratum corneum and membranes and were objects of investigation of the group of Neubert. They irradiated model skin lipid systems with UV light and identified peroxidation products with ESI-MS [49]. Douki et al. and Zhang used HPLC-ESI-MS for the detection of photoproducts in isolated DNA [50,51].

MALDI is a widely used technique for the ionisation of large molecules like proteins. The group of Hillenkamp analysed double stranded DNA by UV- and IR-MALDI [52]. Dreisewerd et al. investigated extracted collagen forming proteins from calfskin using IR-MALDI [53]. However, in these experiments MALDI was used for the detection of components in biological tissues, but not for UV light induced lesions.

\subsection{Direct detection of UV lesions in biological tissue}

Since 1999 several applications for MALDI-MS imaging are developed for this purpose in which the surface is scanned following a certain grid by the exciting laser and mass spectra of every location are obtained [54-59]. In these papers the organic material was analysed either directly or blotted on a MALDI target prepared with C18 beads or on a polymeric conductive membrane. After sample application on the target, a MALDI matrix, which absorbs light at the wavelength of the laser, must be deposited on the sample. As mentioned already, the MALDI matrix should co-crystallise with the analyte, which facilitates desorption and ionisation of the target molecules. Since the atmospheric pressure MALDI technique was developed in 2000 by Laiko et al. there is a possibility to investigate original tissue, too $[60,61]$. As already mentioned in Section 2.4.2 in this context the IR laser is the laser of choice.

Another possibility for the direct analysis of surfaces of biological material using MS was established by Takats et al. in 2004 [62,63], which they called DESI. The aim of this technique is to minimise sample preparation by spraying the object with an electrically charged stream of aqueous micro-droplets. Ions are transported together with the electrospray into the atmospheric pressure interface of the mass spectrometer and analysed there. 


\subsection{Quantification}

Quantitative approaches via mass spectrometry are mostly combined with a chromatographic separation method. Zhang used HPLC-ESI-MS/MS for the quantification of the main photoproducts of DNA in model systems [51]. Tape stripping is a widely used method for the extraction of compounds from living skin of volunteers: the tape removes cell layers together with the substances of interest. Via HPLC-MS it is possible to identify the analyte and to determine its distribution over a certain skin depth. Weigmann et al. showed this for the penetration of the drug clobetasol-propionate [64]. The behaviour of sunscreens applied on human skin was investigated using tape stripping, too [65,66]. However, to our knowledge, until now tape stripping in combination with MS was not used for the analysis of UV generated molecular changes within skin with or without sunscreens.

Quantification using MALDI-MS has its own difficulty due to possible variations in the cocrystallisation of analyte and internal standard with matrix molecules. However, Sleno and Volmer recently quantified marine toxins by MALDI-MS and achieved results comparable to HPLC-ESI-MS/MS experiments [67].

\section{Combination of EPR and MS}

Taking all the discussed aspects together it becomes clear, that the parallel usage of EPR and MS provides the opportunity to combine the results to an overall picture of the investigated system. Whereas the non-invasive EPR imaging gives spatially resolved spectra, which contain information about a stable free radical, its chemical vicinity, and concentration, MALDI provides data about the nature of paraand diamagnetic species of the same system. However, the parallel use of EPR and MS is mentioned only in few reports (see below) and according to our knowledge there are no applications of the direct combination of both methods.

Basic understandings why and how unpaired electrons respond to energy changes within the EPR spectrometer exist, but molecular changes proceeding with a stable free radical inside a mass spectrometer are not thoroughly investigated. Metzger et al. investigated stable free radicals in APCI- and ESI-MS and found them to produce molecular ions $[\mathrm{M}]^{+},[\mathrm{M}+1]^{+\bullet}$ or $[\mathrm{M}+2]^{+}$as the base peak in the mass spectrum depending on their chemical structure, solvent and settings of mass spectrometer [1].

A well-known problem of spin trapping is the unwanted complex interaction of untrapped radical, spin trap, and the resulting adduct. That means, the further reaction of a paramagnetic spin adduct with another radical produces a diamagnetic species, which is EPR silent and can lead to an underestimation of original radical concentration when measured by EPR. Thus, most researchers working in this field first identified the spin trap adducts and their further decomposition products via mass spectrometry [68-72]. Qian et al. presented a method for the identification and quantification of spin-trapped radicals using LC-EPR and HPLC-MS [73].

In order to obtain more information about X-ray induced radicals in a polymer both a spin trap and a spin probe were incorporated simultaneously into the material [74]. A detailed qualitative analysis of products was carried out using GC-MS and EPR. Wright and English compared the method of spin scavenging (using a nitroxide) with that of spin trapping (using a spin trap) for the detection of proteinbased radicals [75]. For this purpose they used MALDI- and ESI-MS and found the efficiency of radical scavenging dramatically higher than that of spin trapping.

However, to our knowledge the combination of EPR and mass spectrometry concerning the investigation of UV induced skin lesions is rarely used. Trommer et al. studied the qualitative molecular changes 
in lipid model systems when radical reactions occur (initiated by a Fenton reaction or UV irradiation) [76,77]. The metabolism of nitroxides in human keratinocytes was studied by Kroll et al. [20]. The aim of our work is to detect and quantify molecular changes within human skin biopsies caused by UV irradiation. Stable nitroxide radicals whose chemistry is simple will be used as reporter molecules [27].

\section{Imaging}

If EPR and MALDI-MS imaging experiments are carried out on skin biopsies one has to consider that skin is a water containing sample resulting in dielectric losses. It attenuates the EPR signal in dependence of its amount and the orientation in the cavity. Nevertheless, the properties of the skin sample, especially the water content, should be maintained during the time of examination, particularly during the time consuming tomography experiments. Furthermore, we preferred AP-MALDI systems to vacuum instruments in order to preserve the samples' nature as good as possible. Moreover, the usage of wet matrices requires the application of an atmospheric pressure ion source and a fast examination of the sample prevents molecular changes during co-crystallisation. The choice of the laser wavelength should be adapted to the analytical question avoiding competing effects between sample treatment and excitation of the molecule for MALDI-MS experiments.

A reproducible sample positioning in the EPR imaging experiment is necessary for the quantification of the stable free radical within the skin. We measure the sample versus an internal DPPH standard whose spin number was quantified using UV-VIS spectroscopy. When MALDI-MS is used for every thin slice of a horizontally cut skin biopsy in the same experiment the proportion of the free radical to the corresponding hydroxylamine can be determined. Thus, the molecular changes of our reporter molecules over skin thickness after UV irradiation are detectable.

Several researchers have contributed to the development of the EPR imaging method [78]. More recently, the group of Zweier has done further work with respect to the instrumental development of new equipment and evaluation procedures for multidimensional EPR imaging in the fields of medicine and biology [79-81]. Nevertheless, there are many investigations described in the literature, which are based on 1D spectral-spatial EPR imaging. That method is characterised by a simple experimental arrangement (one set of gradient coils only) and its reasonable time for recording the necessary set of projections, which is required for image reconstruction.

In order to obtain comparable results using MALDI-MS two approaches are possible: Firstly, the sample is cut into horizontal slices by means of a microtome to get the desired information about the skin layers. Secondly, the method of MALDI imaging explores vertical slices of the skin (see Fig. 9) and the laser spot follows a certain grid to get the spatial information.

It should be pointed out that EPR imaging examines a sample as a body whereas MALDI-MS requires slices of the sample, which is a massive treatment of the biological material.

The MALDI approaches have both advantages and shortcomings. Horizontal cuts supply an average over a certain layer as a result of examining the sample by several less focussed laser spots. The horizontal preparation of the sample is advantageous for adding the matrix, but is more time-consuming. The analysis of vertical cuts (MALDI imaging) is somewhat faster due to the smaller number of slices for measuring. Sample preparation requires the addition of the matrix, which may lead to averaging the layer peculiarities. The method uses a large number of small laser spots to evaluate the sample area, thus reducing the danger that the characteristic nature of the sample is not registered. 


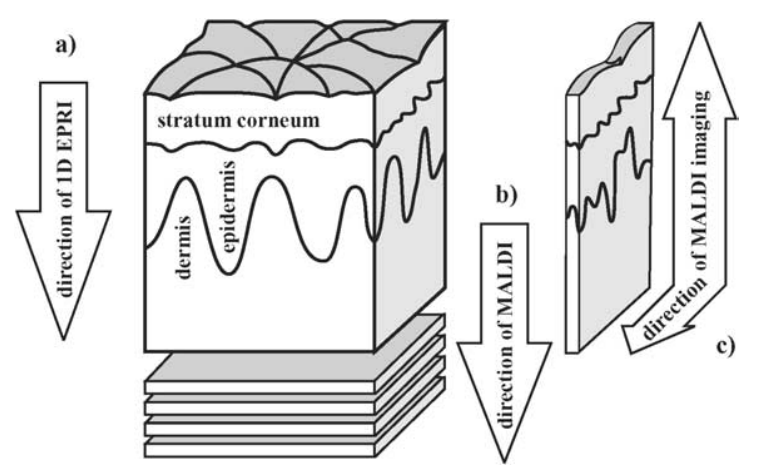

Fig. 9. Imaging in EPR and MALDI-MS. (a) The radical concentration across the skin layers is measured by EPRI non-invasively; (b) for comparable MALDI-MS experiments, the sample has to be cut in horizontal slices; (c) for MALDI imaging, at least one vertical cut is needed.

\section{Summary}

From the discussion, it is evident that MS and EPR are powerful techniques for the detection of UV induced molecular changes within human skin. Until now, only a few studies have been published with parallel applications of both methods. The reasons are obvious: Due to the low stability, which means very short lifetime of the generated primary radicals, they are not directly detectable neither by EPR nor by any MS approach. Furthermore, the analysis of intact tissue samples with mass spectrometric techniques became possible only recently and is still far from reliable or routine.

The following list points to the most relevant issues to be considered when UV damages in skin are object of investigations by MS and EPR in parallel:

- Reproducible preparation and viability of skin biopsies.

- Stabilisation of UV induced radicals for direct detection by spin trapping, or using their reactions with spin probes of known structure and subsequent chemistry.

- Behaviour of reporter molecules within biological materials considering Nernst distributions, molecular dynamics, and chemical transformations.

- The chemistry of the reporter molecule under irradiation and under the influence of the mass spectrometer.

- Imaging conditions in EPR and MALDI-MS (choice of laser excitation and matrix in MALDI experiment).

- Appropriate internal standard for quantification.

However, the recent developments, some of them indicated in this review, provide a new basis for the combination of both analytical methods. The synergistic results that is data on the quantity and distribution of radicals in tissue samples on the one hand and data on the chemical modifications and lesion induced in skin compartments on the other may allow to get new insight in the importance of the different types of UV light. They may shed new light on the real role of sunscreens and should allow measuring the action of such products much more accurately as before.

\section{References}

[1] J.O. Metzger and J. Griep-Raming, Electrospray ionization and atmospheric pressure ionization mass spectrometry of stable organic radicals, Eur. Mass Spectrom. 5 (1999), 157-163. 
[2] R. Marks, The stratum corneum barrier: The final frontier, in: WALTHAM International Science Symposium: Nature, Nurture, and the Case for Nutrition, American Society for Nutritional Sciences, 2004, Suppl., pp. 2017S-2021S.

[3] R.C. Wester, J. Christoffel, T. Hartway, N. Poblete, H.I. Maibach and J. Forsell, Human cadaver skin viability for in vitro percutaneous absorption: Storage and detrimental effects of heat-separation and freezing, Pharm. Res. 15 (1998), 82-84.

[4] K.-P. Moll, Mobilität von ESR-Spinsonden nach epikutaner Applikation unter besonderer Berücksichtigung der Wechselwirkungen zwischen Liposomen und der Haut, PhD thesis, Inst. f. Pharmazie, Humboldt-Universität zu Berlin, 2004.

[5] Commission Internationale De L'Éclairage (CIE). Recommendations for the integrated irradiance and the spectral distribution of simulated solar radiation for testing purposes, Technical Report Publication CIE No. 20, 1972.

[6] J.A. Parrish, R.R. Anderson, F. Urbach and D. Pitts, UVA: Biological Effects of Ultraviolet Radiation with Emphasis on Human Responses to Longwave Ultraviolet, Plenum Press, New York, 1978.

[7] R. Glaser, ed., Biophysik, 4th edn, Gustav Fischer Verlag, 07745 Jena, 1996.

[8] B. Forslind, M. Lindberg, G.M. Roomans, J. Pallon and Y. Werner-Linde, Aspects on the physiology of human skin: Studies using particle probe analysis, Microscopy Research and Technique 38 (1997), 373-386.

[9] S. Tornaletti and G.P. Pfeifer, UV damage and repair mechanisms in mammalian cells, BioEssays 18 (1996), 221-228.

[10] G.P. Pfeifer, Y.-H. You and A. Besaratinia, Mutations induced by ultraviolet light - review, Mut. Res. 571 (2004), $298-308$.

[11] Y. Matsumura and N. Ananthaswamy, Toxic effects of ultraviolet radiation on the skin - review, Toxicol. Appl. Pharmcol. 195 (2004), 298-308.

[12] F.R. de Gruijl, H.J. van Kranen and L.H.F. Mullenders, UV-induced DNA damage, repair, mutations and oncogenic pathways in skin cancer, J. Photochem. Photobiol. B 63 (2001), 19-27.

[13] A.R. Young, Chromophores in human skin, Phys. Med. Biol. 42 (1997), 789-802.

[14] J. Cadet, T. Douki, D. Gasparutto and J.-L. Ravanat, Oxidative damage to DNA: formation, measurement and biochemical features, Mut. Res. 531 (2003), 5-23.

[15] COLIPA, International sun protection factor (SPF) test method, 2003.

[16] Australian standard AS 2604, 1993.

[17] Deutsche Industrienorm DIN 67502, Charakterisierung der UVA-Schutzwirkung von dermalen Sonnenschutzmitteln durch Transmissionsmessungen unter Berücksichtigung des Lichtschutzfaktors, Technical report, 2005.

[18] L. Zastrow, L. Ferrero, T. Herrling and N. Groth, Integrated sun protection factor: A new sun protection factor based on free radicals generated by UV irradiation, Skin Pharmacol. Physiol. 17 (2004), 219-231.

[19] H.M. Swartz, Principles of the metabolism of nitroxides and their implications for spin trapping, Free Rad. Res. Comms. 9 (1990), 399-405.

[20] C. Kroll, A. Langner and H.-H. Borchert, Nitroxide metabolism in the human keratinocyte cell line HaCaT, Free Radic. Biol. Med. 26 (1999), 850-857.

[21] C. Kroll, Analytik, Stabilität und Biotransformation von Spinsonden sowie deren Einsatz im Rahmen pharmazeutischtechnologischer und biopharmazeutischer Untersuchungen, $\mathrm{PhD}$ thesis, Inst. f. Pharmazie, Humboldt-Universität zu Berlin, 1999.

[22] N. Kocherginsky and H.M. Swartz, eds, Nitroxide Spin Labels: Reactions in Biology and Chemistry, CRC Press, Boca Raton, 1995.

[23] J.F. Keana, S. Pou and G.M. Rosen, Nitroxides as potential contrast enhancing agents for MRI application: influence of structure on the rate of reduction by rat hepatocytes, whole liver homogenate, subcellular fractions, and ascorbate, Magn. Reson. Med. 5 (1987), 525-536.

[24] H.M. Swartz, M. Sentjurc and N. Kocherginsky, Toxicity and the use of nitroxides as drugs, pp. 175-198, in Kocherginsky and Swartz [22], 1995.

[25] J. Fuchs, N. Groth and T. Herrling, Coutaneous tolerance to nitroxide free radicals in human skin, Free Radic. Biol. Med. 24 (1998), 643-648.

[26] G. He, A. Samouilov, P. Kuppusamy and J.L. Zweier, In vivo imaging of the distribution and metabolism of nitroxide radicals in human skin, J. Magn. Res. 148 (2001), 155-164.

[27] U. Hochkirch, W. Herrmann, R. Stößer, H.-H. Borchert and M.W. Linscheid, The influence of UV light on human skin: An ex vivo study using mass spectrometry and spatially resolved EPR. [83].

[28] C. Kroll, W. Herrmann, R. Stößer, H.-H. Borchert and K. Mäder, Influence of drug treatment on the microacidity in rat and human skin - an in vitro electron spin resonance imaging study, Pharm. Res. 18 (2001), 525-530.

[29] W. Herrmann, R. Stößer, K.-P. Moll and H.-H. Borchert, Rates of rotational diffusion and Heisenberg spin exchange as obtained from cw ESR and ESR tomographic experiments on model systems and human skin, Appl. Magn. Res. 28 (2005), $85-106$.

[30] K.-P. Moll, W. Herrmann, R. Stößer and H.-H. Borchert, Polarity of skin layers in excised human skin - an in vitro electron spin resonance imaging study, in preparation.

[31] S.-X. Yan, X.-Y. Hong, Y. Hu and K.-H. Liao, Tempol, one of nitroxides, is a novel ultraviolet-A1 radiation protector for human skin fibroblasts, J. Dermatol. Sci. 37 (2005), 137-143.

[32] E.F. Bernstein, S.K. Kong, D.B. Brown, B.C. Kwak, T. Takeuchi, F.P. Gasparro and J. Uitto, The nitroxide Tempol affords 
protection against ultraviolet radiation in a transgenic murine fibroblast culture model of cutaneous photoaging, Exp. Dermatol. 10 (2001), 55-61.

[33] E. Damiani, L. Greci, R. Parsons and J. Knowland, Nitroxide radicals protect DNA from damage when illuminated in vitro in the presence of dibenzoylmethane and a common sunscreen ingredient, Free Radic. Biol. Med. 26 (1999), 809-816.

[34] T. Herrling, J. Fuchs, J. Rehberg and N. Groth, UV-induced free radicals in the skin detected by ESR spectroscopy and imaging using nitroxides, Free Radic. Biol. Med. 35 (2003), 59-67.

[35] B.A. Jurkiewicz and G.R. Buettner, Ultraviolet light-induced free radical formation in skin: An electron paramagnetic resonance study, Photochem. Photobiol. 59 (1994), 1-4.

[36] B.A. Jurkiewicz and G.R. Buettner, EPR detection of free radicals in UV-irradiated skin: Mouse versus human, Photochem. Photobiol. 64 (1996), 918-922.

[37] R. Haywood, P. Wardman, R. Sanders and C. Linge, Sunscreens inadequately protect against ultraviolet-A-induced free radicals in skin: Implications for skin aging and melanoma? J. Invest. Dermatol. 121 (2003), 862-868.

[38] P. Jurkovicz, M. Sentjurc, M. Gasperlin, J. Kristl and S. Pecar, Skin protection against ultraviolet induced free radicals with ascorbyl palmitate in microemulsions, Eur. J. Pharm. Biopharm. 56 (2003), 59-66.

[39] L. Zastrow, T. Herrling, L.J. Berliner, L. Ferrero and N. Groth, In vivo measurement of free radicals in human skin, IFSCC Magazine 6 (2003), 295-300.

[40] H. Yanagida, Y. Masubuchi, K. Minagawa, T. Ogata, J.-I. Takimoto and K. Koyama, A reaction kinetics model of water sonolysis in the presence of a spin-trap, Ultrasonics Sonochemistry 5 (1999), 133-139.

[41] S.I. Dikalov, I.A. Kirilyuk, M.A. Voinov and I.A. Grigorev, Quantitative detection of superoxide by cyclic hydroxylamines: Comparison with nitrone spin traps. [83].

[42] N.D. Yordanov, Quantitative EPR spectrometry - "state of the art", Appl. Magn. Res. 6 (1994), 241-257.

[43] N.D. Yordanov and M. Ivanova, The present state of quantitative EPR spectrometry: The results from an international experiment, Appl. Magn. Res. 6 (1994), 333-340.

[44] N.D. Yordanov, B. Mladenova and P. Petkov, Studies on the uncertainties in quantitative EPR estimations due to the construction of the cavities used, Anal. Chim. Acta 435 (2002), 155-162.

[45] V. Nagy, Quantitative EPR: Some of the most difficult problems, Appl. Magn. Res. 6 (1994), 259-285.

[46] H.M. Swartz, N. Khan, J. Buckey, R. Comi, L. Gould, O. Grinberg, A. Hartford, H. Hopf, H. Hou, E. Hug, A. Iwasaki, P. Lesniewski, I. Salikhov and T. Walczak, Clinical applications of EPR: overview and perspectives, NMR Biomed. 17 (2004), 335-351.

[47] M.C. Krishna, P. Kuppusamy, M. Afeworki, J.L. Zweier, J.A. Cook, S. Subramanian and J.B. Mitchell, Development of functional electron paramagnetic resonance imaging, Breast Disease 10 (1998), 209-220.

[48] S.E. Mudiyanselage, M. Hamburger, P. Elsner and J.J. Thiele, Ultraviolet A induces generation of squalene monohydroperoxide isomers in human sebum and skin surface lipids in vitro and in vivo, J. Invest. Dermatol. 120 (2003), 915-922.

[49] H. Trommer, J. Wagner, H. Graener and R.H.H. Neubert, The examination of skin lipid model systems stressed by ultraviolet irradiation in the presence of transition metal ions, Eur. J. Pharm. Biopharm. 51 (2001), 207-214.

[50] T. Douki, M. Court, S. Sauvaigo, F. Odin and J. Cadet, Formation of the main UV-induced thymine dimeric lesions within isolated and cellular DNA as measured by high performance liquid chromatography-tandem mass spectrometry, J. Biol. Chem. 275 (2000), 11678-11685.

[51] G. Zhang, Characterization and quantitative analysis of UV-induced DNA damage using HPLC-MS/MS spectrometry, PhD thesis, Inst. f. Chemie, Humboldt-Universität zu Berlin, 2005.

[52] F. Kirpekar, S. Berkenkamp and F. Hillenkamp, Detection of double-stranded DNA by IR- and UV-MALDI mass spectrometry, Anal. Chem. 71 (1999), 2334-2339.

[53] K. Dreisewerd, A. Rohlfing, B. Spottke, C. Urbanke and W. Henkel, Characterization of whole fibril-forming collagen proteins of types I, III, and V from fetal calf skin by infrared matrix-assisted laser desorption ionization mass spectrometry, Anal. Chem. 76 (2004), 3482-3491.

[54] M.L. Reyzer and R.M. Caprioli, MALDI mass spectrometry for direct tissue analysis: A new tool for biomarker discovery, J. Proteome Res. 4 (2005), 1138-1142.

[55] T.C. Rohner, D. Staab and M. Stoeckli, MALDI mass spectrometric imaging of biological tissue sections, Mechanisms of Ageing and Development 126 (2005), 177-185.

[56] S.A. Schwartz, M.L. Reyzer and R.M. Caprioli, Direct tissue analysis using matrix-assisted laser desorption/ionization mass spectrometry: practical aspects of sample preparation, J. Mass Spec. 38 (2003), 699-708.

[57] B. Spengler and M. Hubert, Scanning microprobe matrix-assisted laser desorption ionization (SMALDI) mass spectrometry: Instrumentation for sub-micrometer resolved LDI and MALDI surface analysis, J. Am. Soc. Mass Spec. 13 (2002), 735-748.

[58] M. Stoeckli, T.B. Farmer and R.M. Caprioli, Automated mass spectrometry imaging with a matrix-assisted laser desorption ionization time-of-flight instrument, J. Am. Soc. Mass Spec. 10 (1999), 67-71.

[59] P. Chaurand, M. Stoeckli and R.M. Caprioli, Direct profiling of proteins in biological tissue sections by MALDI mass spectrometry, Anal. Chem. 71 (1999), 5263-5270. 
[60] V.V. Laiko, S.C. Moyer and R.J. Cotter, Atmospheric pressure MALDI/ion trap mass spectrometry, Anal. Chem. 72 (2000), 5239-5243.

[61] V.V. Laiko, M.A. Baldwin and A.L. Burlingame, Atmospheric pressure matrix-assisted laser desorption/ionization mass spectrometry, Anal. Chem. 72 (2000), 652-657.

[62] Z. Takats, J.M. Wiseman, B. Gologan and G. Cooks, Mass spectrometry sampling under ambient conditions with desorption electrospray ionization, Science (Reports) 306 (2004), 471-473.

[63] Z. Takats, J.M. Wiseman and G. Cooks, Ambient mass spectrometry using desorption electrospray ionization (DESI): instrumentation, mechanisms and applications in forensics, chemistry, and biology, J. Mass Spec. 40 (2005), 1261-1275.

[64] H.-J. Weigmann, J. Lademann, R.V. Pelchrzim, W. Sterry, T. Hagemeister, R. Molzahn, M. Schaefer, M. Linscheid, H. Schaefer and V.P. Shah, Bioavailability of clobetasol propionate - quantification of drug concentrations in the stratum corneum by dermatopharmacokinetics using tape stripping, Skin Pharmacol. Appl. Skin Physiol. 12 (1999), 46-53.

[65] E. Chatelain, B. Gabard and C. Surber, Skin penetration and sun protection factor of five UV filters: Effect of the vehicle, Skin Pharmacol. Appl. Skin Physiol. 16 (2003), 28-35.

[66] U. Jacobi, H.-J. Weigmann, M. Baumann, A.-I. Reiche, W. Sterry and J. Lademann, Lateral spreading of topically applied UV filter substances investigated by tape stripping, Skin Pharmacol. Physiol. 17 (2004), 17-22.

[67] L. Sleno and D.A. Volmer, Toxin screening in phytoplankton: Detection and quantitation using MALDI triple quadrupole mass spectrometry, Anal. Chem. 77 (2005), 1509-1517.

[68] M.E. Trudeau-Lame, A.S. Kalgutkat and M. LaFontaine, Pharmacokinetics and metabolism of the reactive oxygen scavenger $\alpha$-phenyl-n-tert-butylnitrone in the male sprague-dawley rat, Anal. Chem. 31 (2003), 147-152.

[69] S.Y. Qian, Q. Guo and R.P. Mason, Identification of spin trapped carbon-centered radicals in soybean lipoxygenasedependent peroxidations of $\omega$-3-polyunsaturated fatty acids by LC/ESR, LC/MS, and tandem MS, Free Radic. Biol. Med. 35 (2003), 33-44.

[70] L.J. Deterding, D.C. Ramirez, J.R. Dubin, R.P. Mason and K.B. Tomer, Identification of free radicals on hemoglobin from its selfperoxidation using mass spectrometry and immuno-spin trapping, J. Biol. Chem. 279 (2004), 11600-11607.

[71] H. Iwahashi, C.E. Parker, R.P. Mason and K.B. Tomer, Combined liquid chromatography/electron paramagnetic resonance spectrometry/electrospray ionization mass spectrometry for radical identification, Anal. Chem. 64 (1992), 2244-2252.

[72] Q. Guo, S.Y. Qian and R.P. Mason, Separation and identification of DMPO adducts of oxygen-centered radicals formed from organic hydroperoxides by HPLC-ESR, ESI-MS and MS/MS, J. Am. Soc. Mass Spectrom. 14 (2003), 862-871.

[73] S.Y. Qian, M.B. Kadiiska, Q. Guo and R.P. Mason, A novel protocol to identify and quantify all spin trapped free radicals from in vitro/in vivo interaction of $\mathrm{HO}^{\bullet}$ and DMSO: LC/ESR, LC/MS, and dual spin trapping combinations, Free Radic. Biol. Med. 38 (2005), 125-135.

[74] B. Bittner, K. Mäder, C. Kroll, H.-H. Borchert and T. Kissel, Tetracycline-HCl-loaded poly(DL-lactide-co-glycolide) microspheres prepared by a spray drying technique: influence of $\gamma$-irradiation on radical formation and polymer degradation, J. Controlled Release 59 (1999), 23-32.

[75] P.J. Wright and A.M. English, Scavenging with TEMPO $\bullet$ to identify peptide- and protein-based radicals by mass spectrometry: Advantages of spin scavenging over spin trapping, J. Am. Chem. Soc. 125 (2003), 8655-8665.

[76] H. Trommer, R. Böttcher, A. Pöppl, J. Hoentsch, S. Wartewig and R.H.H. Neubert, Role of ascorbic acid in stratum corneum lipid models exposed to UV irradiation, Pharm. Res. 19 (2002), 982-990.

[77] H. Trommer, S. Wartewig, R. Böttcher, A. Pöppl, J. Hoentsch, J.H. Ozegowski and R.H.H. Neubert, The effects of hyaluronan and its fragments on lipid models exposed to UV irradiation, Int. J. Pharm. 254 (2003), 223-234.

[78] G.R. Eaton, S.S. Eaton and K. Ohno, eds, EPR Imaging and In Vivo EPR, CRC Press Inc., Boca Raton, 1991.

[79] P. Kuppusamy, M. Chzhan and J.L. Zweier, 3D and 4D electron paramagnetic resonance imaging of the rat heart, Magn. Res. Med. 6 (1995), 59-61.

[80] P. Kuppusamy, M. Chzhan and J.L. Zweier, Development and optimization of three-dimensional spatial EPR imaging for biological organs and tissues, J. Magn. Res. B 106 (1995), 122-130.

[81] P. Kuppusamy, M. Chzhan, A. Samouilov, P. Wang and J.L. Zweier, Mapping the spin-density and lineshape distribution of free radicals using 4D spectral-spatial EPR imaging, J. Magn. Res. B 107 (1995), 116-125.

[82] N.D. Yordanov and A.G. Christova, Quantitative spectrophotometric and EPR-determination of 1,1-diphenyl-2-picrylhydrazyl (DPPH), Fresenius J. Anal. Chem. 358 (1997), 610-613.

[83] Novosibirsk Institute of Organic Chemistry, International Tomography Center, Institute of Chemical Kinetics and Cobumstion, Novosibirsk State University, 4th International Conference on Nitroxide Radicals: Synthesis, Properties and Implications of Nitroxides (SPIN-2005), 2005. 


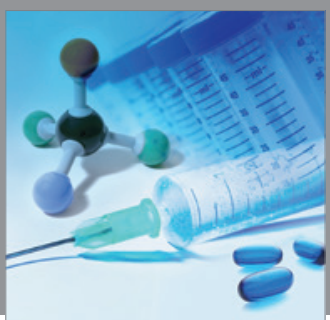

International Journal of

Medicinal Chemistry

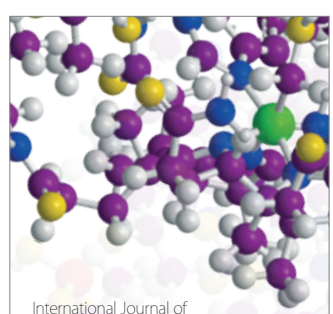

Carbohydrate Chemistry

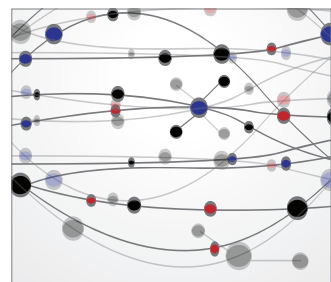

The Scientific World Journal
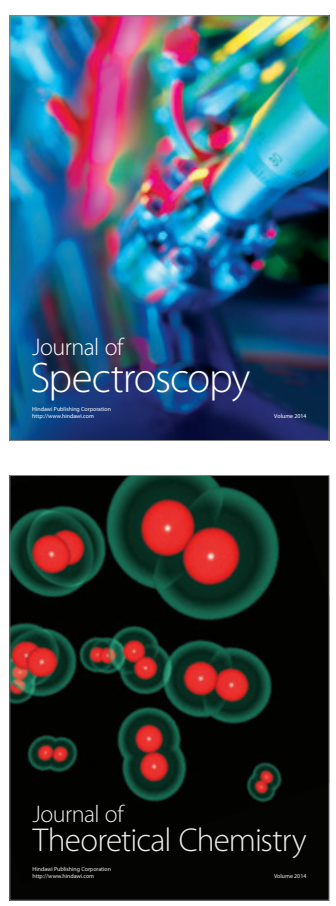
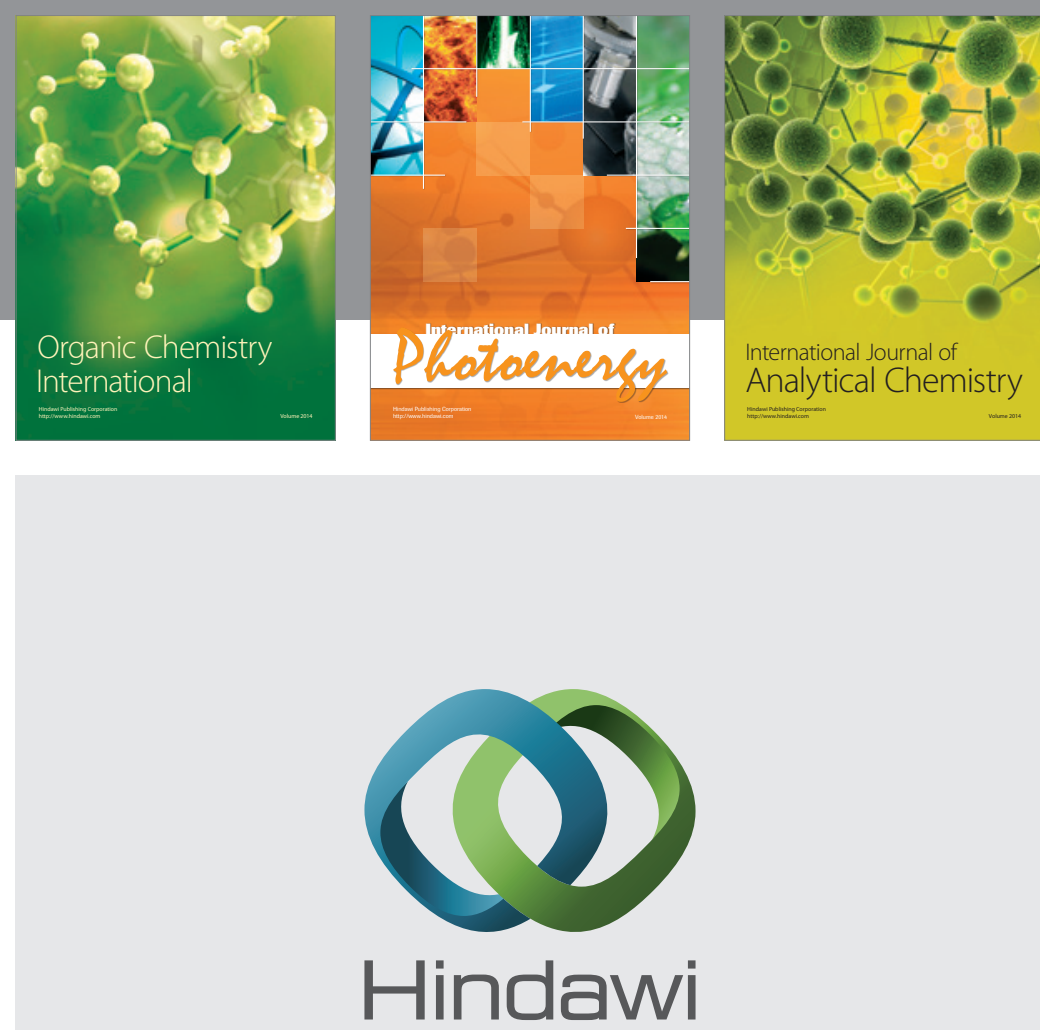

Submit your manuscripts at

http://www.hindawi.com
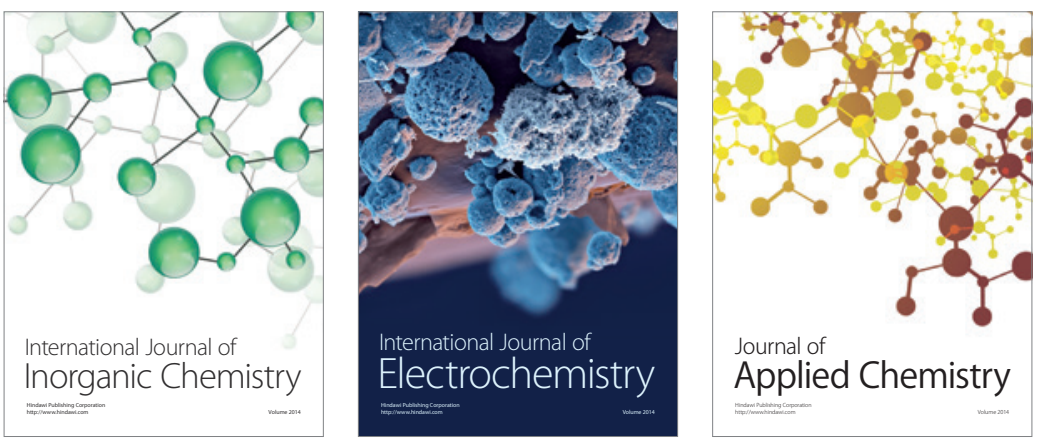

Journal of

Applied Chemistry
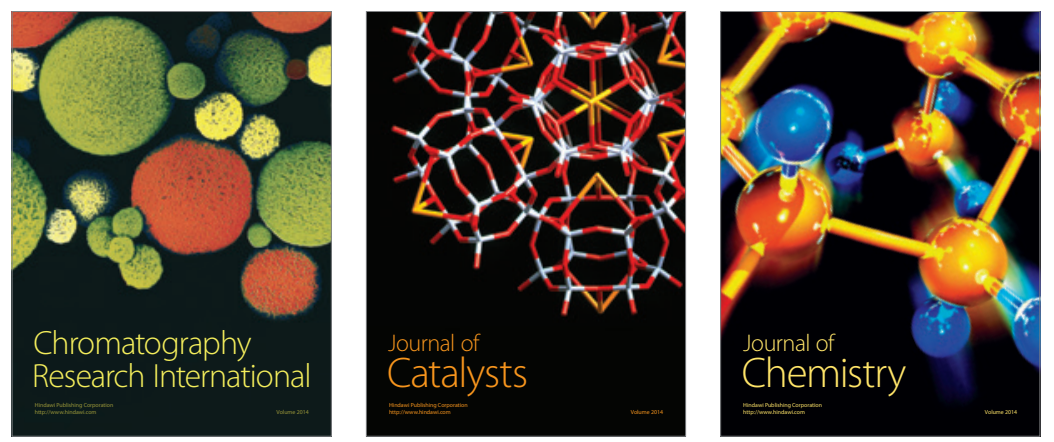
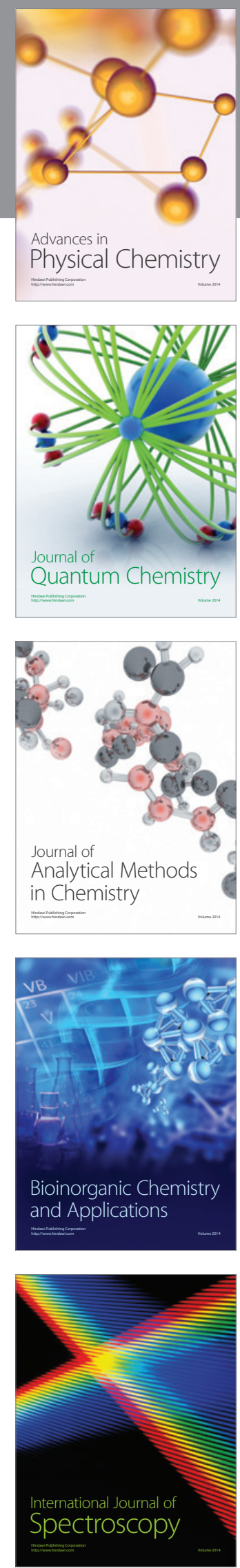